\title{
Therapeutic effect of carbon monoxide-releasing molecule-3 on acute lung injury after hemorrhagic shock and resuscitation
}

\author{
YUTA KUMADA $^{1}$, TORU TAKAHASHI ${ }^{2}$, HIROKO SHIMIZU ${ }^{1}$, RYU NAKAMURA ${ }^{1}$, \\ EMIKO OMORI ${ }^{1}$, KAZUYOSHI INOUE $^{3}$ and HIROSHI MORIMATSU ${ }^{1}$ \\ ${ }^{1}$ Department of Anesthesiology and Resuscitology, Okayama University Graduate School of Medicine, \\ Dentistry and Pharmaceutical Sciences, Okayama 700-8558; ${ }^{2}$ Faculty of Health and Welfare Science, \\ Okayama Prefectural University, Soja, Okayama 719-1197; ${ }^{3}$ Department of Anesthesiology, \\ Kagawa Prefectural Central Hospital, Takamatsu, Kagawa 760-8557, Japan
}

Received August 28, 2018; Accepted February 11, 2019

DOI: $10.3892 /$ etm.2019.7390

\begin{abstract}
Hemorrhagic shock and resuscitation (HSR) induces a pulmonary inflammatory response and frequently causes acute lung injury. Carbon monoxide-releasing molecule-3 (CORM-3) has been reported to liberate and deliver CO under physiological conditions, which exerts organ-protective effects during systemic insults. The present study aimed to determine whether the administration of CORM-3 following HSR exerts a therapeutic effect against HSR-induced lung injury without any detrimental effects on oxygenation and
\end{abstract}

Correspondence to: Dr Yuta Kumada, Department of Anesthesiology and Resuscitology, Okayama University Graduate School of Medicine, Dentistry and Pharmaceutical Sciences, 2-5-1, Shikatacho, Okayama-shi, Okayama 700-8558, Japan

E-mail: blackbear.kssy@gmail.com

Abbreviations: ABG, arterial blood gas; ARDS, acute respiratory distress syndrome; BAL, bronchoalveolar lavage; BE, base excess; BKCa channels, large conductance calcium-activated potassium channels; $\mathrm{CO}$, carbon monoxide; $\mathrm{COHb}$, carboxyhemoglobin; CORMs, carbon monoxide-releasing molecules; CORM-3, carbon monoxide-releasing molecule-3; ELISA, enzyme-linked immunosorbent assay; $\mathrm{Hb}$, hemoglobin; $\mathrm{HO}$, heme oxygenase; $\mathrm{HO}-1$, heme oxygenase-1; HSR, hemorrhagic shock and resuscitation; iCORM-3, inactive carbon monoxide-releasing molecule-3; IL-10, interleukin-10; IL-1 $\beta$, interleukin-1 $\beta$; iNOS, inducible nitric oxide synthase; MAPK, mitogen-activated protein kinases; MIP-2, macrophage inflammatory protein-2; NF- $\mathrm{B}$, nuclear factor $\kappa \mathrm{B}$; NLRP3, nucleotide-binding domain, leucine-rich-containing family, pyrin domain-containing-3; $\mathrm{PaCO}_{2}$, partial pressure of carbon dioxide; $\mathrm{PaO}_{2}$, partial pressure of oxygen; RT-qPCR, reverse transcription-quantitative polymerase chain reaction; $\mathrm{SaO}_{2}$, saturation of arterial blood oxygen; SEM, standard error of the mean; TNF- $\alpha$, tumor necrosis factor- $\alpha$; TUNEL, transferase-mediated dUTP-fluorescein isothiocyanate (FITC) nick-end labeling

Key words: acute lung injury, inflammation, carbon monoxide, carbon monoxide-releasing molecule-3, hemorrhagic shock and resuscitation hemodynamics. To induce hemorrhagic shock, rats were bled to a mean arterial blood pressure of $30 \mathrm{mmHg}$ for $45 \mathrm{~min}$ and then resuscitated with the shed blood. CORM-3 or a vehicle was intravenously administered immediately following the completion of resuscitation. The rats were divided into four groups, including sham, HSR, HSR/CORM-3 and HSR/inactive CORM-3 groups. Arterial blood gas parameters and vital signs were recorded during HSR. The histopathological changes to the lungs were evaluated using a lung injury score, while pulmonary edema was evaluated on the basis of the protein concentration in bronchoalveolar lavage fluid and the lung wet/dry ratio. We also investigated the pulmonary expression levels of inflammatory mediators and apoptotic markers such as cleaved caspase- 3 and transferasemediated dUTP-fluorescein isothiocyanate nick-end labeling (TUNEL) staining. Although HSR caused significant lung histopathological damage and pulmonary edema, CORM-3 significantly ameliorated this damage. CORM-3 also attenuated the HSR-induced upregulation of tumor necrosis factor- $\alpha$, inducible nitric oxide synthase and interleukin- $1 \beta$ genes, and the expression of interleukin-1 $\beta$ and macrophage inflammatory protein-2. In addition, the expression of interleukin-10, an anti-inflammatory cytokine, was inversely enhanced by CORM-3, which also reduced the number of TUNEL-positive cells and the expression of cleaved caspase- 3 following HSR. Although CORM-3 was administered during the acute phase of HSR, it did not exert any influence on arterial blood gas analysis data and vital signs during HSR. Therefore, treatment with CORM-3 ameliorated HSR-induced lung injury, at least partially, through anti-inflammatory and anti-apoptotic effects, without any detrimental effects on oxygenation and hemodynamics.

\section{Introduction}

Hemorrhagic shock and resuscitation (HSR) is known to induce a pulmonary inflammatory response that leads to acute lung injury, which is referred to as acute respiratory distress syndrome (ARDS) (1-4). Although lung protective ventilation has been proposed for the treatment of ARDS in clinical 
settings $(5,6)$, there is no definitive pharmacological therapy to prevent pulmonary inflammation in ARDS (6-8).

Carbon monoxide $(\mathrm{CO})$ is widely known to be a toxic gaseous molecule that produces carboxyhemoglobin $(\mathrm{COHb})$ due to its higher affinity to hemoglobin $(9,10)$. However, small amounts of $\mathrm{CO}$ are endogenously produced by the enzymatic reaction of heme oxygenase (HO) that degrades heme to biliverdin, iron (II) ion, and CO. In addition, heme oxygenase-1 (HO-1), the inducible isoform of $\mathrm{HO}$ that is rapidly induced by a vast array of stress stimuli, plays an important role in organ protection by degrading free heme, a prooxidant, and producing CO $(11,12)$. Recent findings have indicated that exogenous inhalation of low-dose concentrations of $\mathrm{CO}$ conferred cytoprotective effects against insults in various animal models of inflammatory disorders (13-17). Our previous study also demonstrated that inhalation of $\mathrm{CO}$ at $250 \mathrm{ppm}$ significantly ameliorated the HSR-induced acute lung injury via its anti-inflammatory and anti-apoptotic properties (18). However, even this low dose of CO inhalation therapy elevated arterial $\mathrm{COHb}$ levels by approximately $20 \%$ (19), which may be toxic to humans (20). To avoid the adverse effect of $\mathrm{CO}$ inhalation, carbon monoxide-releasing molecules (CORMs) have been developed by coordinating $\mathrm{CO}$ with transition metal carbonyl complexes, which spontaneously liberate and deliver $\mathrm{CO}$ to organ tissues under physiological conditions through all possible routes of administration (21-24). CORMs have also been reported to exert cytoprotective effects against various animal models of oxidative damage without altering the blood $\mathrm{COHb}$ levels (22-28). However, to our knowledge, few studies have focused on the effect of CORMs on HSR-induced acute lung injury, although a previous report showed the beneficial effect of CORM-3 [tricarbonylchloro-glycinate ruthenium(II) ( $\mathrm{Ru}(\mathrm{CO}) 3 \mathrm{Cl}$-glycinate); a water-soluble form of CORMs] on HSR-induced liver injury (29). Moreover, the influence of CORMs on arterial oxygenation and hemodynamics during the acute phase of HSR remains to be elucidated.

Thus, in the present study, we investigated whether CORM-3 administration immediately after resuscitation exerted a therapeutic effect on HSR-induced lung injury in a rat HSR model without showing any detrimental influence on oxygenation and hemodynamics.

\section{Materials and methods}

Animals. This study was approved by the Animal Use and Care Committee of the Okayama University Medical School (OKU-2015429) on September 2, 2015 and conformed to the guidelines for the care and use of laboratory animals that followed the ARRIVE (Animal Research: Reporting of In Vivo Experiments) guidelines (30) and the 2013 AVMA euthanasia guidelines (31). Male Sprague-Dawley rats weighing 370 to $430 \mathrm{~g}$ were purchased from Clea Japan, Inc. (Tokyo, Japan). The rats were housed in temperature-controlled rooms at $25^{\circ} \mathrm{C}$ with 12 -h light/dark cycles and allowed free access to water and chew until the start of experiments. The total number of rats was 117 in this study; sham group $(n=29)$, HSR group $(n=27)$, HSR/CORM-3 group ( $\mathrm{n}=38)$, and HSR/inactive CORM-3 (iCORM-3) group $(n=23)$.
Preparation of drugs. Water-soluble CORM-3 was purchased from Sigma-Aldrich Japan Inc. (Tokyo, Japan). CORM-3 was solubilized in distilled water $(20 \mathrm{mg} / \mathrm{ml}$ stock $)$ and stored at $-20^{\circ} \mathrm{C}$ until the experiments. iCORM-3, an inactive counterpart of CORM-3, was prepared by incubating CORM-3 in a phosphate-buffered solution ( $\mathrm{pH} 7.4)$ at room temperature for 2 days to liberate all of the $\mathrm{CO}$ gas from the molecule before the experiment (32). At the time of administration, the stock solution was diluted four-fold with sterile saline to make a $5 \mathrm{mg} / \mathrm{ml}$ final concentration of CORM-3 and iCORM3. Then, $4 \mathrm{mg} / \mathrm{kg}$ of CORM-3 or iCORM-3 (0.8 ml/ $\mathrm{kg}$ of CORM-3 or iCORM-3 solution) was intravenously administered through the left femoral vein.

Protocol for HSR. Rats were anesthetized with intraperitoneal sodium pentobarbital $(50 \mathrm{mg} / \mathrm{kg})$ injection and subjected to the HSR procedure as previously described $(33,34)$. In brief, the left inguinal artery and vein were dissected using aseptic techniques, and heparinized polyethylene catheters were inserted into the left femoral vessels. The left femoral artery catheter was used to monitor the arterial blood pressure during the HSR procedure while the left femoral vein catheter was used to withdraw or return the shed blood. Hemorrhagic shock was induced by collecting blood into a heparinized syringe (10 units/ml) from the left femoral vein over 15 min until a mean arterial blood pressure of $30 \mathrm{mmHg}$ was achieved. This pressure level was maintained for an additional $45 \mathrm{~min}$ by further blood withdrawal, after which the rats were resuscitated by reinfusing all the shed blood over $15 \mathrm{~min}$. After a 45-min post-reinfusion monitoring period, the surgical incision was closed. The rats in the sham group underwent all surgical procedures except bleeding. The animals were allowed to breathe spontaneously without tracheal intubation throughout the experiment. All procedures were performed on a heating pad that could perform automatic regulation of rectal body temperature within the physiological range.

Experimental design. To examine the effects of CORM-3 treatment on HSR-induced lung injury, the rats subjected to HSR were randomly assigned to the following groups based on the treatment at the end of resuscitation: The HSR/CORM-3 group was administered $4 \mathrm{mg} / \mathrm{kg}$ of CORM-3; the HSR/iCORM-3 group was administered $4 \mathrm{mg} / \mathrm{kg}$ of iCORM-3; and the HSR group was vehicle-treated (same amount of normal saline as CORM-3 or iCORM-3). Drugs were intravenously administered through the left femoral vein immediately after returning all the shed blood. At specific time points following resuscitation, rats were euthanized by exsanguination from the left femoral artery under inhaled anesthesia by ethyl ether with spontaneous breathing. The lungs were excised and frozen immediately in liquid nitrogen and stored at $-80^{\circ} \mathrm{C}$ until further use. For histological examinations, the right upper lobe of the lung was fixed in $10 \%$ neutral buffered formalin and embedded in paraffin.

Arterial blood gas analysis. Arterial blood samples were drawn from the left femoral artery catheter at the following time points during HSR: $0 \mathrm{~min}$, at the beginning of the hemorrhagic shock; $60 \mathrm{~min}$, at the end of the hemorrhagic shock; 
$80 \mathrm{~min}$, at $5 \mathrm{~min}$ after the completion of the blood reinfusion and the subsequent drug administration; and $120 \mathrm{~min}$, at the end of the monitoring phase after resuscitation. Arterial blood gas (ABG) analysis was performed with ABL80 CO-OX (Radiometer Medical ApS, Copenhagen, Denmark).

Histopathological examination. For histological examinations, the right upper lobe of the lungs was excised $12 \mathrm{~h}$ after resuscitation and fixed in $10 \%$ neutral buffered formalin, embedded in paraffin, and sectioned at a thickness of $5 \mu \mathrm{m}$. After deparaffinization and dehydration, the sections were stained with hematoxylin and eosin for microscopic examination. Lung histological changes were assessed on a light microscope by three observers in a blinded fashion, in accordance with previously described methods (35-37) with modifications for five independent experiments. In brief, a total of 10 randomly selected areas from each lung section were graded separately as 0 (no findings or normal), 1 (mild), 2 (moderate), or 3 (severe) for each of the following four parameters: Congestion, pulmonary edema, cellular infiltration, and hemorrhage. The sum of the individual scores for each parameter was calculated as the lung injury score.

Terminal deoxynucleotidyl transferase-mediated dUTP-fluorescein isothiocyanate nick-end labeling staining. Transferase-mediated dUTP-fluorescein isothiocyanate (FITC) nick-end labeling (TUNEL) staining was performed using the MEBSTAIN Apoptosis TUNEL Kit Direct (No. 8445; MBL, Nagano, Japan) according to the manufacturer's instructions. Sections were incubated with terminal deoxynucleotidyl transferase and FITC-conjugated dUTP, followed by counterstaining with $0.5 \mu \mathrm{g} / \mathrm{ml}$ propidium iodide. The number of TUNEL-positive cells was counted in five nonconsecutive sections per rat at a magnification of $x 400$ in a blinded manner with a Zeiss confocal laser scanning microscope model LSM510 (Zeiss, Jena, Germany).

Lung wet weight to dry weight (wet/dry) ratio. Left lung tissue samples obtained $12 \mathrm{~h}$ after resuscitation were weighed (wet weight) and dried at $110^{\circ} \mathrm{C}$ for $24 \mathrm{~h}$. Then, the dry tissue weight was measured, and the wet/dry weight ratio was determined as an indicator of pulmonary edema by dividing the lung wet weight by the lung dried weight $(19,34,38)$.

Measurement of protein concentration in the bronchoalveolar lavage fluid. Protein concentration in the bronchoalveolar lavage (BAL) fluid was measured at $6 \mathrm{~h}$ after HSR. Rats were subjected to euthanasia and tracheotomy at $6 \mathrm{~h}$ after HSR, followed by lavage via the tracheal tube with $5 \mathrm{ml}$ of saline (39). The collected BAL fluids were centrifuged at $240 \mathrm{x} \mathrm{g}$ for $15 \mathrm{~min}$ at $4^{\circ} \mathrm{C}$, and the supernatants were stored at $-30^{\circ} \mathrm{C}$ until measurement. Protein concentrations in the BAL fluids were determined using the Pierce ${ }^{\mathrm{TM}}$ BCA Protein Assay Kit (Pierce, Rockford, IL, USA) according to the manufacturer's instructions. The absorbance was measured with a microplate reader, Multiskan JX (Thermo Fisher Scientific, Inc., Yokohama, Japan).

RNA isolation and northern blot analysis. Total RNA was isolated from lung tissues at $3 \mathrm{~h}$ after HSR by using TRI
REAGENT $^{\circledR}$ (Molecular Research Center, Inc., Cincinnati, $\mathrm{OH}$, USA) according to the manufacturer's instructions. Northern blot analysis was performed as described previously $(40,41)$. In brief, $20 \mu \mathrm{g}$ of total RNA was subjected to electrophoresis in $1.2 \%(\mathrm{w} / \mathrm{v})$ agarose gel containing $6.5 \%$ (v/v) formaldehyde and transferred to a Bio-Rad Zeta-Probe GT Blotting Membrane (Bio-Rad Laboratories, Richmond, CA, USA). The blotted membrane was hybridized with [ $\alpha-32 \mathrm{P}]$ dCTP-labeled cDNA probes for tumor necrosis factor (TNF)- $\alpha$ and inducible nitric oxide synthase (iNOS), respectively, followed by rigorous washing. The membrane was exposed to a sheet of Fuji Medical X-ray film (Fujifilm Co., Tokyo, Japan) with an intensifying screen at $-70^{\circ} \mathrm{C}$. Autoradiographs were quantified with an image scanner (ChemiDoc $^{\mathrm{TM}}$ XRS plus; Bio-Rad Laboratories, Tokyo, Japan) and computerized image analysis software (Image $\mathrm{Lab}^{\mathrm{TM}}$ version 5.0; Bio-Rad Laboratories, Tokyo, Japan). The relative amounts of radiolabeled cDNA that hybridized to the blots were normalized to the levels of $18 \mathrm{~S}$ ribosomal RNA for correction of loading errors.

Reverse transcription-quantitative polymerase chain reaction $(R T-q P C R)$. Total RNA was extracted from lung tissues as described above. Purification of total RNA was performed using the RNeasy ${ }^{\circledR}$ Mini kit (Qiagen Sciences, Germantown, MD, USA). After removal of potentially contaminating DNA with DNase I (RNase-Free DNase set; Qiagen GmbH, Hilden, Germany), reverse transcription of total RNA was performed by using a QuantiTect ${ }^{\circledR}$ Reverse Transcription Kit (Qiagen $\mathrm{GmbH}$, Hilden, Germany) to generate first-strand cDNA. The PCR reaction mixture was prepared by using $\mathrm{SYBR}^{\circledR}$ Premix Ex Taq ${ }^{\mathrm{TM}}$ (Takara Bio Inc., Shiga, Japan). The PCR was performed using a LightCycler (Roche Diagnostics $\mathrm{GmbH}$, Mannheim, Germany), as previously described (42). The sequences of the upstream and downstream primers for interleukin-10 (IL-10), interleukin-1 $\beta$ (IL-1 $\beta$ ), and $\beta$-actin were as follows: 5'-ACGCTGTCATCGATTTCTC-3' and 5'-GGCCTT GTAGACACCTTTG-3' for IL-10; 5'-AGCTATGGCAACTGT CCCTGAA-3' and 5'-CATCTGGACAGCCCAAGTCAA for IL-1 $\beta$; 5'-AACCCTAAGGCCAACCGTGAA-3' and 5'-CAG GGACAACACAGCCTGGA-3' for $\beta$-actin, respectively. The mRNA levels of IL-10 and IL-1 $\beta$ were normalized to the mRNA level of $\beta$-actin.

Protein extraction and measurement of protein expression by enzyme-linked immunosorbent assay (ELISA). To extract protein from lung tissue, lung homogenates at $12 \mathrm{~h}$ after HSR were prepared as previously described with minor modifications (43). In brief, $0.4 \mathrm{~g}$ of lung samples were mixed with $1 \mathrm{ml}$ of a phosphate-buffered saline $-0.1 \%$ Triton-X100 solution containing a protease inhibitor cocktail (cOmplete; Roche Diagnostics Indianapolis, IN, USA), homogenized, and centrifuged at $23,800 \mathrm{x}$ g for $15 \mathrm{~min}$ at $4^{\circ} \mathrm{C}$. The supernatants were collected and stored for subsequent analysis. The protein levels of IL-1 $\beta$ and macrophage inflammatory protein-2 (MIP-2) in the lung homogenates were determined by an ELISA kit (R\&D Systems ${ }^{\circledR}$, Minneapolis, MN, USA) according to the manufacturer's instructions. All assays were performed in duplicate. Data are presented as the amount per gram of fresh weight of lung tissue. 
Western blot analysis. Western blot analysis was performed as described previously (44). The protein concentrations of lung homogenates were determined using the Pierce $\mathrm{BCA}^{\mathrm{TM}}$ Protein Assay Kit (Pierce, Rockford, IL, USA). Samples equivalent to $50 \mu \mathrm{g}$ were applied to 12.5 or $15 \%$ (w/v) polyacrylamide-SDS gels. After electrophoretic separation, the proteins were transferred to 'Amersham' Hybond-polyvinylidene fluoride membranes (GE Healthcare Japan Co., Tokyo, Japan). The membrane was blocked with Tris-buffered saline containing 4\% (w/v) BlockAce ${ }^{\mathrm{TM}}$ (DS Pharma Biomedical Co., Ltd., Osaka, Japan) for $1 \mathrm{~h}$ at room temperature, followed by incubation with the primary antibody against cleaved caspase-3 (rabbit anti-cleaved caspase-3 [Asp175] polyclonal antibody: 9661S, Cell Signaling, 1:500 dilution) or GAPDH (rabbit anti-GAPDH [FL-335] polyclonal antibody: sc-25778, SANTA CRUZ, 1:5,000 dilution) for $20 \mathrm{~h}$ at $4^{\circ} \mathrm{C}$. After washing the membranes with buffer, the membranes were incubated with the secondary antibody (goat anti-rabbit IgG-HRP: sc-2004; Santa Cruz, 1:20,000 dilution) for $30 \mathrm{~min}$ at room temperature. The membranes were stained with Clarity Western ECL Substrate (Bio-Rad, USA) according to the manufacturer's instructions. Antigen-antibody complexes were visualized with an image scanner (ChemiDoc XRS Plus Imaging System, Bio-Rad) and the intensity of signals was quantified by using an analysis software (Image Lab Version 5.0, Bio-Rad).

Statistical analysis. Data are expressed as mean \pm standard error of the mean (SEM). Statistical analyses were performed using analysis of variance followed by the Tukey-Kramer multiple comparisons method, as appropriate. A two-sided $\mathrm{P}<0.05$ was considered to indicate a statistically significant difference. Comparisons were examined by using JMP Pro $12^{\circledR}$ software (SAS Institute Inc., Cary, NC, USA).

\section{Results}

Effect of CORM-3 on HSR-induced histological damage in the lung. First, we assessed the effect of CORM-3 on HSR-induced histological damage in the lungs $12 \mathrm{~h}$ after resuscitation by performing microscopic examinations of lung sections stained with hematoxylin and eosin (magnification, x400). Lung sections of the HSR group revealed prominent histopathological changes, including inflammatory cell infiltration, alveolar septal thickening, interstitial edema, and hyaline membrane formation, while those of the sham group appeared almost normal. In contrast, CORM-3 treatment following HSR markedly improved these histopathological changes, although iCORM-3 administration did not affect the pathological findings caused by HSR (Fig. 1A). These findings were confirmed by the lung injury score, which was used to evaluate histopathological changes related to congestion, edema, inflammation, and hemorrhage in a blinded fashion. The lung injury scores in rats of the HSR group were significantly higher than those in the sham group, but CORM-3 treatment significantly reduced the score to levels almost identical to those in sham-operated rats (Fig. 1B).

Effect of CORM-3 on lung wet/dry ratio and the protein concentration in the BAL fluid. Since the lung histological examination showed HSR-induced pulmonary edema and its improvement by CORM-3, we further evaluated the lung wet/dry ratio and the protein concentration in the BAL fluid as indicators of lung edema and vascular permeability, respectively. Consistent with the histological findings, the lung wet/dry ratio at $12 \mathrm{~h}$ after HSR in the HSR group was markedly higher than that in the sham group (Fig. 1C). In contrast, CORM-3 treatment following HSR significantly suppressed the HSR-induced increase in the lung wet/dry ratio. However, the ratio in the HSR/iCORM-3 group was as high as that in the HSR group. Furthermore, we also found that the protein concentration in the BAL fluid in HSR animals was significantly higher (approximately 3-fold higher) than that in sham-operated animals (Fig. 1D). Consistent with the observations for the wet/dry ratio, only CORM-3 treatment significantly reduced the protein concentration. These results, together with the histopathological findings, indicate that CORM-3 treatment ameliorated lung edema and the HSR-induced increase in pulmonary vascular permeability.

Effect of CORM-3 on gene expressions of proinflammatory mediators in the lung. CORM-3 treatment significantly suppressed the HSR-induced infiltration of inflammatory cells to the lung in histological examinations. Therefore, in order to clarify the anti-inflammatory effect of CORM-3, we examined the effect of CORM-3 on the gene expressions of inflammatory mediators in the lung. We measured the gene expression levels of the proinflammatory mediators TNF- $\alpha$ and iNOS by northern blot analysis and that of IL- $1 \beta$ by RT-qPCR using lung samples obtained $3 \mathrm{~h}$ after HSR. While the mRNA levels in the sham group were relatively low, those in the HSR group had significantly increased (Fig. 2A-C). In contrast, CORM-3 treatment markedly reduced the increased expression levels observed in the HSR group to less than half of the mRNA levels in the HSR group, although the HSR/iCORM-3 group showed no change in those mRNA levels in comparison with the levels in the HSR group (Fig. 2A-C).

Effect of CORM-3 on the expressions of proinflammatory mediators in the lung. Next, we measured the protein expression levels of inflammatory cytokines such as IL-1 $\beta$ and MIP-2 in the lung homogenates at $12 \mathrm{~h}$ following HSR by ELISA. The expression levels of these cytokines were markedly higher in the HSR group than in the sham group (Fig. 3A and B). In contrast, CORM-3 treatment significantly reduced the elevated expression levels of these cytokines to almost the same levels as those observed in the sham group, while iCORM-3 did not show any influence on the HSR-induced upregulation of these inflammatory cytokines (Fig 3A and B).

Effect of CORM-3 on the gene expression of IL-10, an anti-inflammatory cytokine, in the lung. To further investigate the anti-inflammatory effects of CORM-3, we examined the gene expression of IL-10, an anti-inflammatory cytokine, by RT-qPCR in the lungs at $3 \mathrm{~h}$ after HSR. Although pulmonary gene expression of IL-10 mRNA was barely detectable in the sham group, it was increased by the HSR procedure. Notably, the CORM-3 treatment group showed drastically upregulated IL-10 mRNA levels compared to those in the other three groups (Fig. 2D). 
A

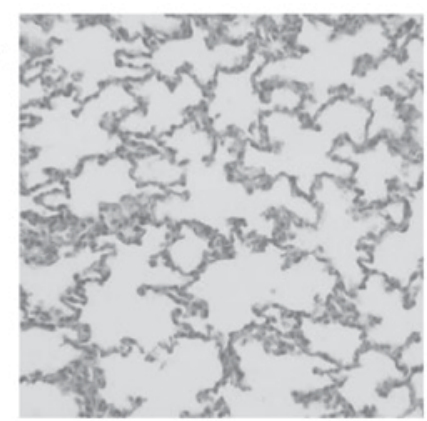

Sham

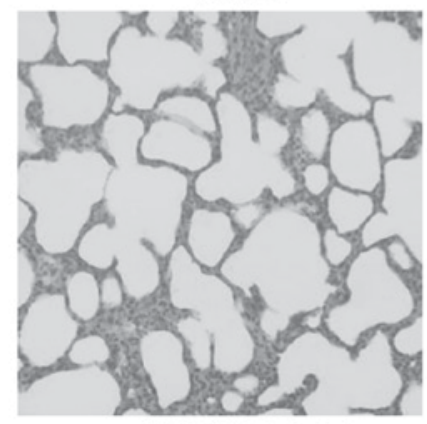

HSR/CORM-3

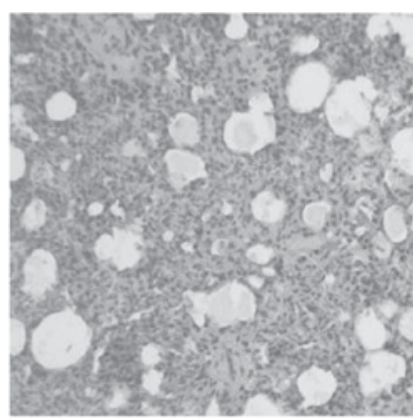

HSR

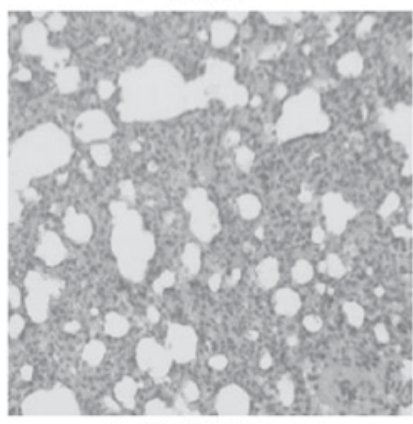

HSR/iCORM-3
B

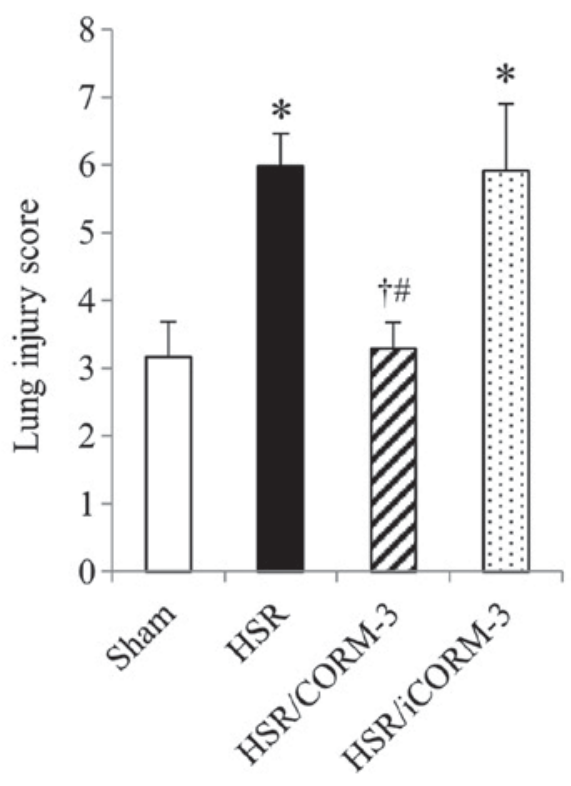

C

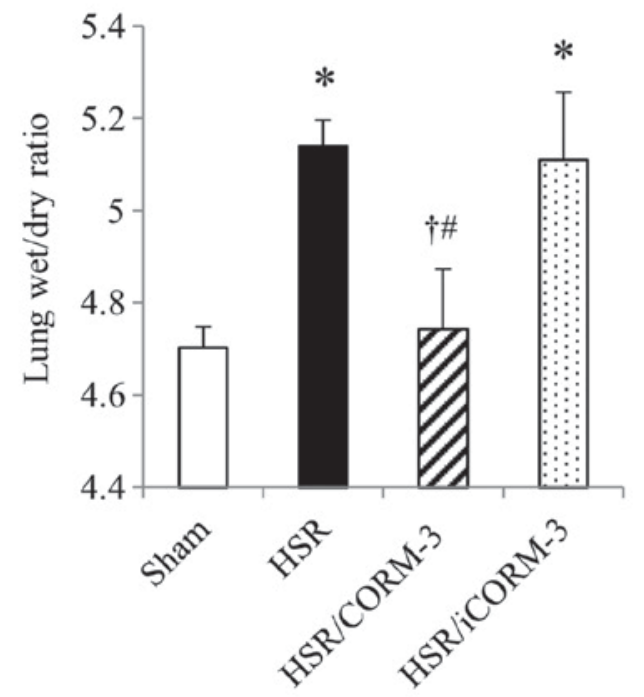

D $(\mu \mathrm{g} / \mathrm{ml})$

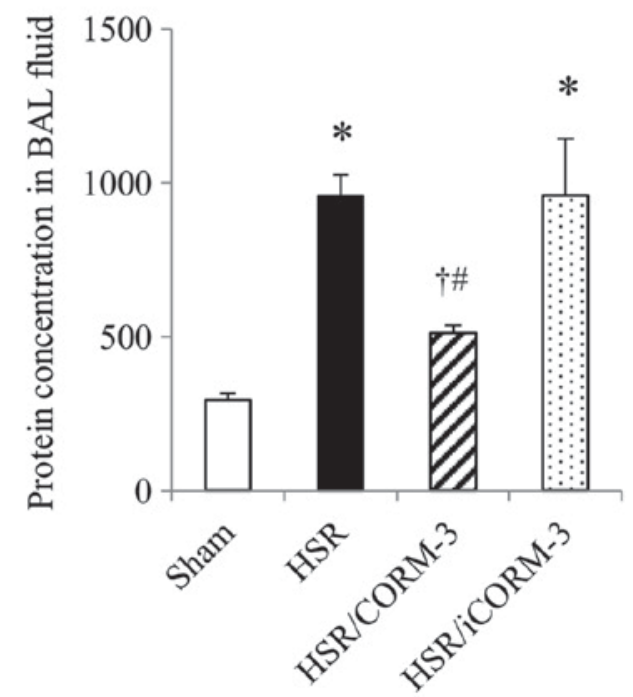

Figure 1. Effect of CORM-3 on HSR-induced lung histological damage, lung wet/dry ratio, and the protein concentration in the BAL fluid. Lungs were excised $12 \mathrm{~h}$ following resuscitation and stained with hematoxylin and eosin for microscopic examination. Lung wet/dry ratio and protein concentration in the BAL fluid were evaluated at $6 \mathrm{~h}$ following HSR. (A) Representative images from five independent experiments (hematoxylin-eosin staining; original magnification, $\mathrm{x} 400$ ). (B) Lung injury score; the severity of histopathological changes in the lungs $12 \mathrm{~h}$ following HSR was assessed by three independent observers. Ten areas randomly selected from each lung section were graded separately as 0 (no findings or normal), 1 (mild), 2 (moderate), or 3 (severe) for each of the following four parameters: Congestion, pulmonary edema, cellular infiltration, and hemorrhage. The sum of these four scores was calculated as the lung injury score. (C) Lung wet/dry ratio. Excised left lungs at $12 \mathrm{~h}$ following HSR were weighed (wet weight) and dried at $110^{\circ} \mathrm{C}$ for $24 \mathrm{~h}$ (dry weight). The wet/dry ratio was calculated by dividing the lung wet weight by the dry weight. (D) Protein concentration in the BAL fluid. BAL fluid was collected by lavage with $5 \mathrm{ml}$ of saline via a tracheotomy at $6 \mathrm{~h}$ after HSR. Statistical analysis was performed using analysis of variance followed by Tukey-Kramer multiple comparisons method. Data in each analysis are presented as the mean \pm SEM ( $\mathrm{n}=5$ for each group). ${ }^{*} \mathrm{P}<0.05$ (sham vs. HSR, sham vs. HSR $/ \mathrm{iCORM}-3$ ), ${ }^{\dagger} \mathrm{P}<0.05$ (HSR vs. HSR/CORM-3), and " $\mathrm{P}<0.05$ (HSR/CORM-3 vs. HSR/iCORM-3). BAL, bronchoalveolar lavage; CORM-3, carbon monoxide-releasing molecule-3; HSR, hemorrhagic shock and resuscitation; iCORM-3, inactive carbon monoxide-releasing molecule-3; SEM, standard error of the mean.

Effect of CORM-3 on apoptotic cell death in the lungs following HSR. Pulmonary ischemia reperfusion induces tissue inflammation, which causes oxidative stress and ultimately leads to the apoptosis of alveolar endothelial cells (45). Therefore, we examined the effect of CORM-3 on apoptotic cell death by performing TUNEL staining, which specifically stains the 3'-OH DNA ends generated by DNA fragmentation of apoptosis, and assessed the protein expressions of cleaved caspase- 3 , an executive molecule of apoptosis, in the lungs at $12 \mathrm{~h}$ after HSR. While the lung sections from the 

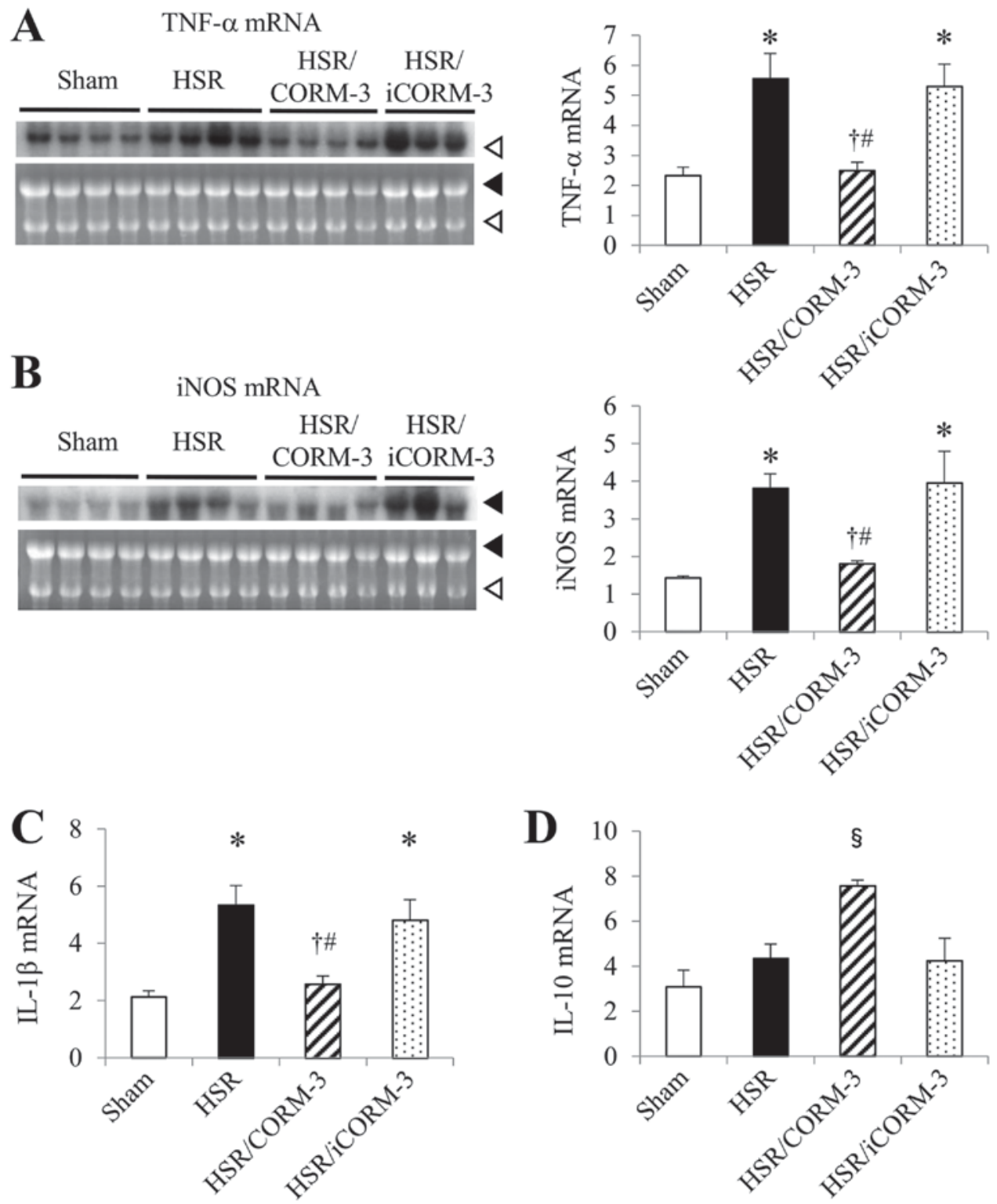

Figure 2. Effect of CORM-3 on gene expressions of pro- and anti-inflammatory mediators in the lung following HSR. Lungs from HSR rats were excised at $3 \mathrm{~h}$ following resuscitation. Then, the mRNA levels of TNF-a and iNOS were measured by northern blot analysis. (A and B) The closed arrowhead indicates $28 \mathrm{~S}$ ribosomal RNA, while the open arrowhead indicates $18 \mathrm{~S}$ ribosomal RNA. The relative amounts of hybridized radiolabeled cDNAs were normalized to 18 S ribosomal RNA levels; mRNA levels of IL-1 $\beta$ (C) and IL-10 (D) were measured by RT-qPCR. Data from RT-qPCR were normalized to $\beta$-actin levels. Statistical analysis was performed using analysis of variance followed by the Tukey-Kramer multiple comparisons method among groups. Data in each analysis are presented as the mean \pm SEM in arbitrary units $\left(n=3-5\right.$ for each group). ${ }^{*} \mathrm{P}<0.05$ (sham vs. HSR, sham vs. HSR $/ \mathrm{iCORM}-3$ ), ${ }^{\circ} \mathrm{P}<0.05$ (HSR vs. HSR/CORM-3), "P<0.05 (HSR/CORM-3 vs. HSR/iCORM-3), and ${ }^{\$} \mathrm{P}<0.05$ (HSR/CORM-3 vs. sham, HSR, and HSR/iCORM-3). CORM-3, carbon monoxide-releasing molecule-3; HSR, hemorrhagic shock and resuscitation; iCORM-3, inactive carbon monoxide-releasing molecule-3; IL-1 $\beta$, interleukin-1 $\beta$; IL-10, interleukin-10; iNOS, inducible nitric oxide synthase; RT-qPCR, reverse transcription-quantitative polymerase chain reaction; SEM, standard error of the mean; TNF- $\alpha$, tumor necrosis factor- $\alpha$.

sham group showed a few TUNEL-positive cells, the HSR procedure resulted in a significant increase in the number of TUNEL-positive cells (Fig. 4A and B). In contrast, CORM-3 treatment significantly reduced the number of TUNEL-positive cells to approximately one-fourth of the HSR group, although the HSR/iCORM-3 group showed an increase in the number of TUNEL-positive cells to the same extent as that noted in the HSR group. Consistent with the changes observed in TUNEL staining, the level of cleaved caspase- 3 expression was significantly elevated in the HSR group, while it was barely detectable in the sham group (Fig 4C and D). Conversely, CORM-3 treatment significantly suppressed the upregulation of cleaved caspase- 3 to one-third of that in the HSR group, whereas iCORM-3 administration did not exert any effect on the expression of cleaved caspase-3 (Fig. 4C and D). These findings indicate that CORM-3 treatment significantly attenuated HSR-induced lung injury by its anti-apoptotic effect.

CORM-3 administration has no influence on the hemodynamic status during HSR. Since CORM-3 has been reported to have a vasodilatory effect through the activation of soluble guanylate cyclase and large conductance calcium-activated potassium channels (BKCa channels) $(21,32,46)$, we examined the effect of CORM-3 treatment on the hemodynamic 

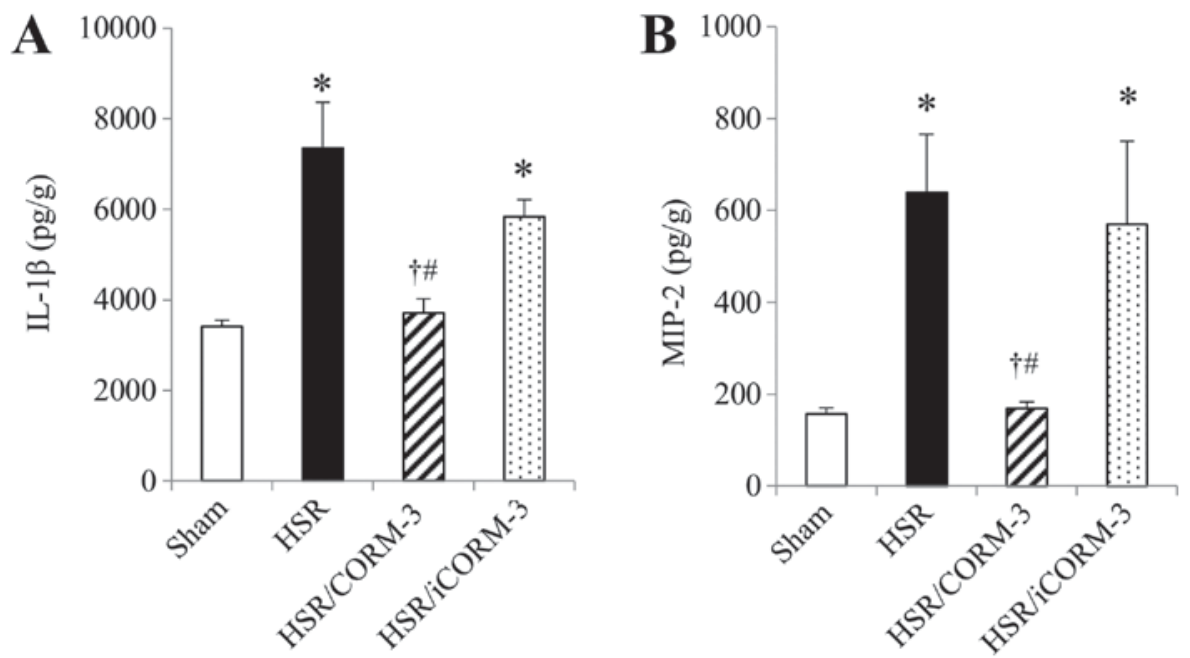

Figure 3. Effect of CORM-3 on protein expressions of HSR-induced inflammatory mediators in the lungs following HSR. Lungs from HSR rats were excised and homogenized at $12 \mathrm{~h}$ following resuscitation. Pulmonary protein expressions of inflammatory cytokines were measured by an enzyme-linked immunosorbent assay. (A) Interleukin-1 $\beta$ (IL-1 $\beta$ ) levels in lung homogenates at $12 \mathrm{~h}$ following HSR. (B) Macrophage inflammatory protein-2 (MIP-2) levels in lung homogenates at $12 \mathrm{~h}$ following HSR. Values are reported as the amount per gram of lung tissue (pg/g). Statistical analysis was performed using analysis of variance followed by the Tukey-Kramer multiple comparisons method among groups. Data are presented as the mean $\pm \mathrm{SEM}$ ( $\mathrm{n}=4-5$ for each group). ${ }^{*} \mathrm{P}<0.05$ (sham vs. HSR, sham vs. HSR/iCORM-3), ${ }^{\circ} \mathrm{P}<0.05$ (HSR vs. HSR/CORM-3), and ${ }^{\#} \mathrm{P}<0.05$ (HSR/CORM-3 vs. HSR/iCORM-3). CORM-3, carbon monoxide-releasing molecule-3; HSR, hemorrhagic shock and resuscitation; iCORM-3, inactive carbon monoxide-releasing molecule-3; IL-1 $\beta$, interleukin-1 $\beta$; MIP-2, macrophage inflammatory protein-2; SEM, standard error of the mean.

status. Although only the shed blood was returned at the time of resuscitation without any additional fluid requirement in the rats subjected to HSR, the time course changes in mean arterial blood pressure and heart rate in all experimental groups were almost identical throughout the HSR procedure (Fig. 5), indicating that CORM-3 at this dose did not affect the animals' hemodynamic status during HSR.

CORM-3 administration following HSR has no influence on blood $\mathrm{COHb}$ levels and preserves oxygenation as measured by $A B G$ analysis during HSR. Since CORM-3 releases trace amounts of $\mathrm{CO}$ after intravenous administration, its treatment may cause an increase in blood $\mathrm{COHb}$ levels that could lead to derangement of the physiologic homeostasis. Thus, we performed $\mathrm{ABG}$ analysis at four specific time points during HSR, as indicated in the Materials and methods section. Notably, there were no significant differences on the blood $\mathrm{COHb}$ levels among all experimental groups at all time points, and CORM-3 treatment did not affect arterial oxygenation, as indicated by the maintenance of the $\mathrm{PaO}_{2}$ and $\mathrm{SaO}_{2}$ levels (Fig. 6). Although the levels of hemoglobin (Hb), pH, and base excess (BE) at the end of the hemorrhagic shock in all groups subjected to HSR were significantly lower than those in the sham group, probably because of the systemic ischemia and metabolic acidosis induced by the hemorrhagic shock, there were no significant differences in these indices among the groups subjected to HSR. These results indicate that CORM-3 treatment influenced neither blood $\mathrm{COHb}$ levels nor the physiologic homeostasis in the arterial blood.

\section{Discussion}

The present study demonstrated that administration of CORM-3 immediately after the completion of resuscitation following hemorrhagic shock ameliorated the HSR-induced acute lung injury. This fact was ascertained by the improvements in the histological damage and lung edema caused by the increased pulmonary vascular permeability. We also found that CORM-3 treatment significantly decreased the expressions of inflammatory mediators, such as TNF- $\alpha$, iNOS, IL-1 $\beta$, and MIP-2, and increased the expression of IL-10, an anti-inflammatory cytokine. Moreover, CORM-3 administration decreased apoptotic cell death, evidenced by the decrease in TUNEL-positive cells and cleaved caspase-3 expression. In contrast, CORM-3 administration did not influence arterial $\mathrm{COHb}$ levels and oxygenation or exert any detrimental effects on hemodynamic status in HSR animals. These findings indicate that CORM-3 exerts potent therapeutic effects on HSR-induced acute lung injury, which are at least in part mediated through its anti-inflammatory and anti-apoptotic properties.

Hemorrhagic shock and resuscitation causes oxidative stress and produces excess amounts of free heme by the destabilization of hemoproteins, leading to various tissue injuries involving the lung, liver, kidney, and intestine (12). To protect against the insult, the enzymatic reaction of HO-1 is rapidly induced to catalyze the degradation of free heme into biliverdin, iron (II) ion, and CO $(11,12)$. Our group and others have reported that induction of HO-1 ameliorated oxidative tissue injuries induced by HSR, and the deletion or inhibition of HO- 1 conversely exacerbated the injuries $(34,47,48)$. Thus, the HO-1 system is thought to constitute an essential cytoprotective component against HSR-induced oxidative organ damage. Although CO is a byproduct of heme breakdown, the molecule itself has been reported to possess anti-inflammatory and anti-apoptotic properties mediated through several signaling pathways, including p38 mitogen-activated protein kinases (MAPK), nuclear factor $\kappa \mathrm{B}(\mathrm{NF}-\kappa \mathrm{B})$, the nucleotide-binding domain, leucine-rich-containing family, pyrin domain-containing-3 (NLRP3)-dependent inflammasome, and 
A

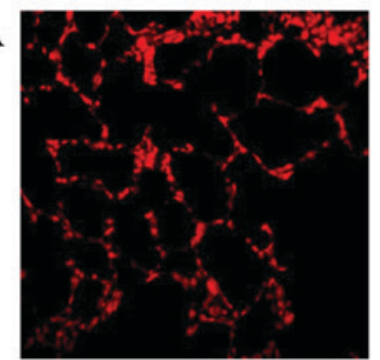

Sham

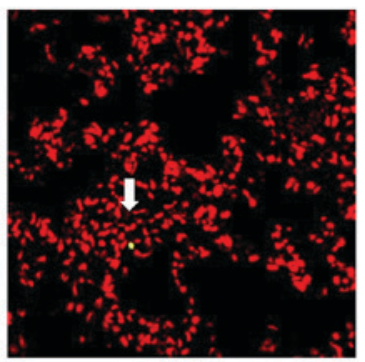

HSR/CORM-3

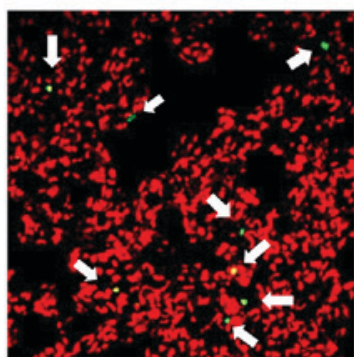

HSR

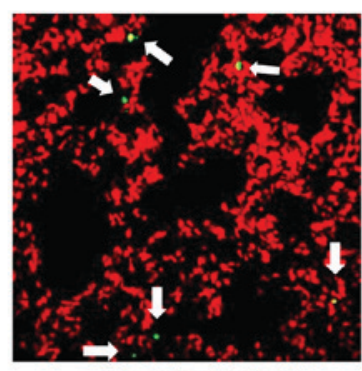

HSR/iCORM-3
C

Cleaved caspase-3

GAPDH
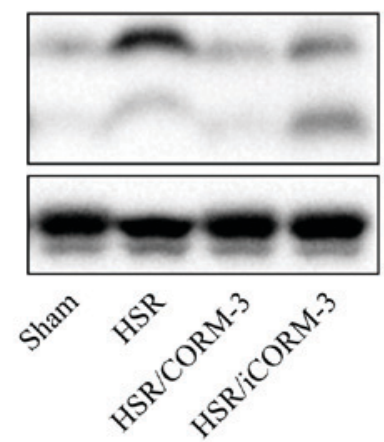

B

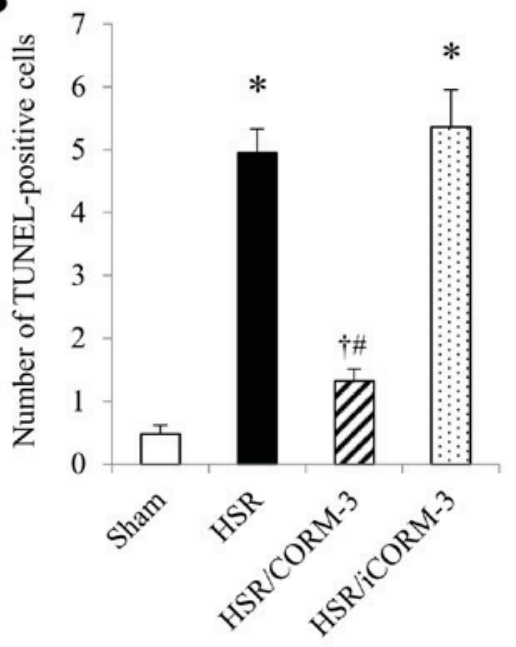

D

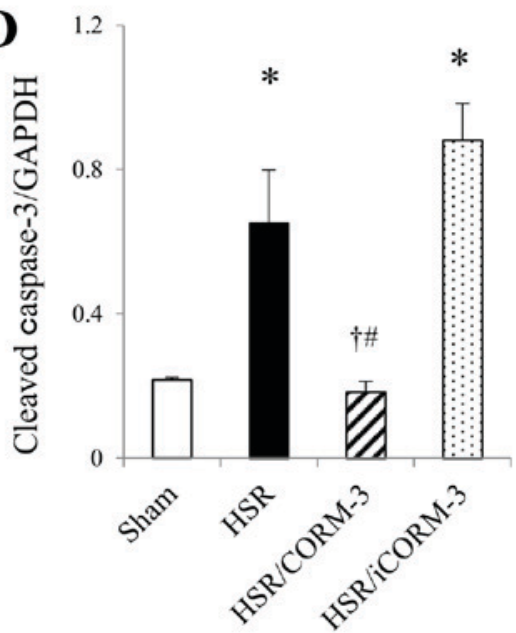

Figure 4. Effect of CORM-3 on apoptotic cell death in the lungs following HSR. Lungs were excised $12 \mathrm{~h}$ following resuscitation and examined for apoptotic cell death by the terminal deoxynucleotidyl transferase-mediated dUTP-fluorescein isothiocyanate (FITC) nick-end labeling (TUNEL) method and by assessment of protein expression of cleaved caspase-3 measured by western blot analysis. (A) Representative images of the lung sections stained by TUNEL method at $12 \mathrm{~h}$ following HSR (x400 original magnification). The white arrows indicate TUNEL-positive cells. (B) The quantification of TUNEL-positive cells in the lung sections at $12 \mathrm{~h}$ following HSR. Data are presented as the mean \pm SEM ( $\mathrm{n}=5$ for each group). (C) Western blot analysis by using an antibody against cleaved caspase-3 and an antibody against GAPDH as the loading control. (D) Densitometric measurement of cleaved caspase-3 normalized to GAPDH levels in western blot analysis. Data are presented as the mean \pm SEM ( $n=3$ for each group). Statistical analysis was performed using analysis of variance followed by the Tukey-Kramer multiple comparisons method among groups. " $\mathrm{P}<0.01$ (sham vs. HSR, sham vs. HSR/iCORM-3), ${ }^{\dagger} \mathrm{P}<0.01$ (HSR vs. HSR/CORM-3), and ${ }^{*} \mathrm{P}<0.01$ (HSR/CORM-3 vs. HSR/iCORM-3). CORM-3, carbon monoxide-releasing molecule-3; HSR, hemorrhagic shock and resuscitation; iCORM-3, inactive carbon monoxide-releasing molecule-3; SEM, standard error of the mean; TUNEL, transferase-mediated dUTP-fluorescein isothiocyanate (FITC) nick-end labeling.

modulation of mitochondrial biogenesis, among others (49-56). CORMs, which release $\mathrm{CO}$, also have been reported to exert a cytoprotective effect against organ injury through the same signaling pathways as CO $(26-28,54,57,58)$. Previous studies for acute lung injury induced by thermal injury or sepsis indicated that CORMs-released CO suppressed NF- $\kappa \mathrm{B}$ activation, thereby decreasing the expression of proinflammatory mediators, including TNF- $\alpha$, IL- $1 \beta$, and NO $(57,58)$. The beneficial effects of CORMs have also been reported to be associated with the upregulation of IL-10, mediated through induction of HO-1, in a p38MAPK-dependent manner (27). CORMs-induced IL-10 expression attenuates activation of the NLRP-3-dependent inflammasome, which exacerbates the inflammatory response by producing IL-1 $\beta$ in sepsis-induced lung injury (54). These findings are consistent with our results in the present study. As for the model of HSR-induced lung injury, our previous studies demonstrated that $\mathrm{CO}$ inhalation therapy at 250 ppm exerted anti-inflammatory and anti-apoptotic effects against HSR-induced lung injury, mediated through the activation of peroxisome proliferator-activated receptor (PPAR) $-\gamma$, or the suppression of NF- $\kappa \mathrm{B}$ and activator protein-1 $(18,19)$. The anti-inflammatory and anti-apoptotic effect of CORM-3 demonstrated in the present study might be involved in those upstream signaling pathways. Although further investigation is needed, the present study is valuable in demonstrating the beneficial effect of CORM-3 against HSR-induced lung injury. As far as we know, few studies have investigated the effect of CORM-3 on acute lung injury 

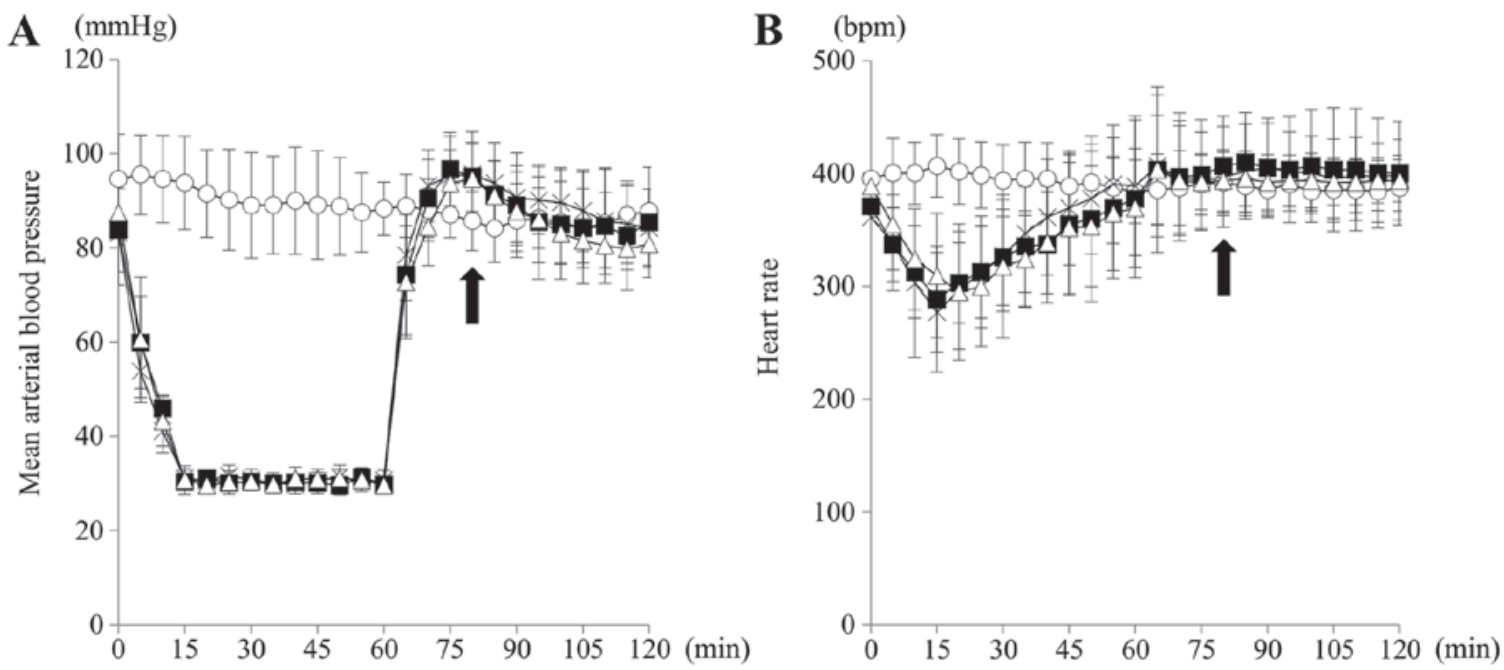

$$
-\mathrm{O} \text { Sham } \leftarrow \text { HSR } \rightarrow \text { HSR/CORM-3 } \leftrightharpoons \text { HSR/iCORM-3 }
$$

Figure 5. CORM-3 administration has no influence on vital signs during HSR. Rats subjected to HSR were administered CORM-3, iCORM-3, or a vehicle (normal saline) through the left femoral vein immediately following the completion of resuscitation. Mean arterial blood pressure and heart rate were measured at 5 min intervals during the HSR procedure. (A) Mean arterial blood pressure at 5-min intervals during HSR. The horizontal axis indicates the time after the beginning of the hemorrhagic shock, and the vertical axis shows the mean arterial blood pressure. The closed arrow indicates the time of the drug or vehicle administration. (B) Heart rate at 5-min intervals during HSR. The horizontal axis indicates the time after the beginning of the hemorrhagic shock, and the vertical axis shows the heart rate. The closed arrow indicates the time of the drug or vehicle administration. Statistical analysis was performed using analysis of variance followed by Tukey-Kramer multiple comparisons method for the mean arterial blood pressure and heart rate at each time point in the HSR, HSR/CORM-3, and HSR/iCORM-3 groups. Data are presented as the mean \pm SEM ( $=10$ for each group). CORM-3, carbon monoxide-releasing molecule-3; HSR, hemorrhagic shock and resuscitation; iCORM-3, inactive carbon monoxide-releasing molecule-3; SEM, standard error of the mean.

induced by HSR. Although Nassour et al demonstrated that CORM-3 reduces systemic inflammation and hepatic sinusoidal endothelial injury after HSR (29), they did not focus on lung injury. Moreover, they administered CORM-3 during the resuscitation period, whereas we administered CORM-3 immediately after completion of resuscitation. Thus, the present study is, to the best of our knowledge, the first study to demonstrate the usefulness of CORM-3 administration post-resuscitation against HSR-induced lung injury.

We previously demonstrated that inhalation of CO at $250 \mathrm{ppm}$ for $3 \mathrm{~h}$ after completion of resuscitation significantly attenuated HSR-induced acute lung injury via the anti-inflammatory and anti-apoptotic properties of CO (18). However, even $1 \mathrm{~h}$ of $\mathrm{CO}$ inhalation at $250 \mathrm{ppm}$ significantly increased blood $\mathrm{COHb}$ levels by up to approximately $20 \%$ in rats $(13,19,49)$, which may exert toxic effects in humans (20). Notably, in the present study, CORM-3 treatment markedly ameliorated HSR-induced acute lung injury without influencing the blood $\mathrm{COHb}$ levels. CORMs are transition metal carbonyl complexes coordinated with $\mathrm{CO}$ that can liberate and deliver $\mathrm{CO}$ to tissues once in contact with suitable physiological conditions (21-24). CORM-3, a water-soluble form of CORMs, rapidly releases CO immediately after administration into lysis buffer ( $\mathrm{t} 1 / 2<1 \mathrm{~min})(23)$. Thus, our findings indicated that intravenous administration of CORM-3 rapidly releases $\mathrm{CO}$ following its incorporation into the lung, which leads to the attenuation of HSR-induced lung injury and inflammation.

CORMs have been reported to have a vasodilatory effect that involves the activation of soluble guanylyl cyclase and BKCa channels (46). According to previous studies, $20-30 \mu \mathrm{mol} / \mathrm{kg}$ of CORM-3 administration reduced the mean arterial blood pressure by approximately $10 \mathrm{mmHg}$ in a normal rat model, and its vasodilatory effect was dosedependent $(21,32)$. However, in the present study, time course changes in mean arterial pressure after resuscitation were not different among the HSR/CORM-3, HSR/iCORM-3, and HSR groups (Fig. 5). The absence of the vasodilatory effect of CORM-3 may be attributable to two factors. First, the dosage of CORM-3 in our study was $4 \mathrm{mg} / \mathrm{kg}(13.58 \mu \mathrm{mol} / \mathrm{kg})$, which was relatively lower than the dosages in previous studies $(21,32)$. Second, as shown in Fig. 2B, the HSR procedure increased the pulmonary expression of iNOS that led to the production of NO, a potent vasodilator. The iNOS gene is expressed in nearly every organ and is associated with refractory hypotension after hemorrhagic shock $(45,59)$. In contrast, CORM-3 treatment markedly decreased the expression of iNOS in the lung in the present study (Fig. 2B). Thus, we speculate that the reduction in iNOS expression with CORM-3 administration may compensate for the vasodilatory effect of CORM-3, although we examined the modulation of iNOS expression by CORM-3 only in the lung. In any case, our findings underscore the safety of CORM-3 treatment with respect to hemodynamic stability, since CORM-3 administration did not show any apparent detrimental influence on vital signs even in the acute, hemodynamically instable state after HSR.

There are several limitations to the present study. First, we did not directly measure the $\mathrm{CO}$ concentration in the pulmonary tissue of our rat HSR model. However, in a pilot study prior to the presented experiments, we confirmed increases in $\mathrm{CO}$ concentrations in the blood and various tissues after CORM-3 administration in healthy control rats (unpublished data). CORM-3 administration significantly increased in a dose-dependent fashion the $\mathrm{CO}$ concentration in lung tissue of these animals. In addition, we administered in the present 

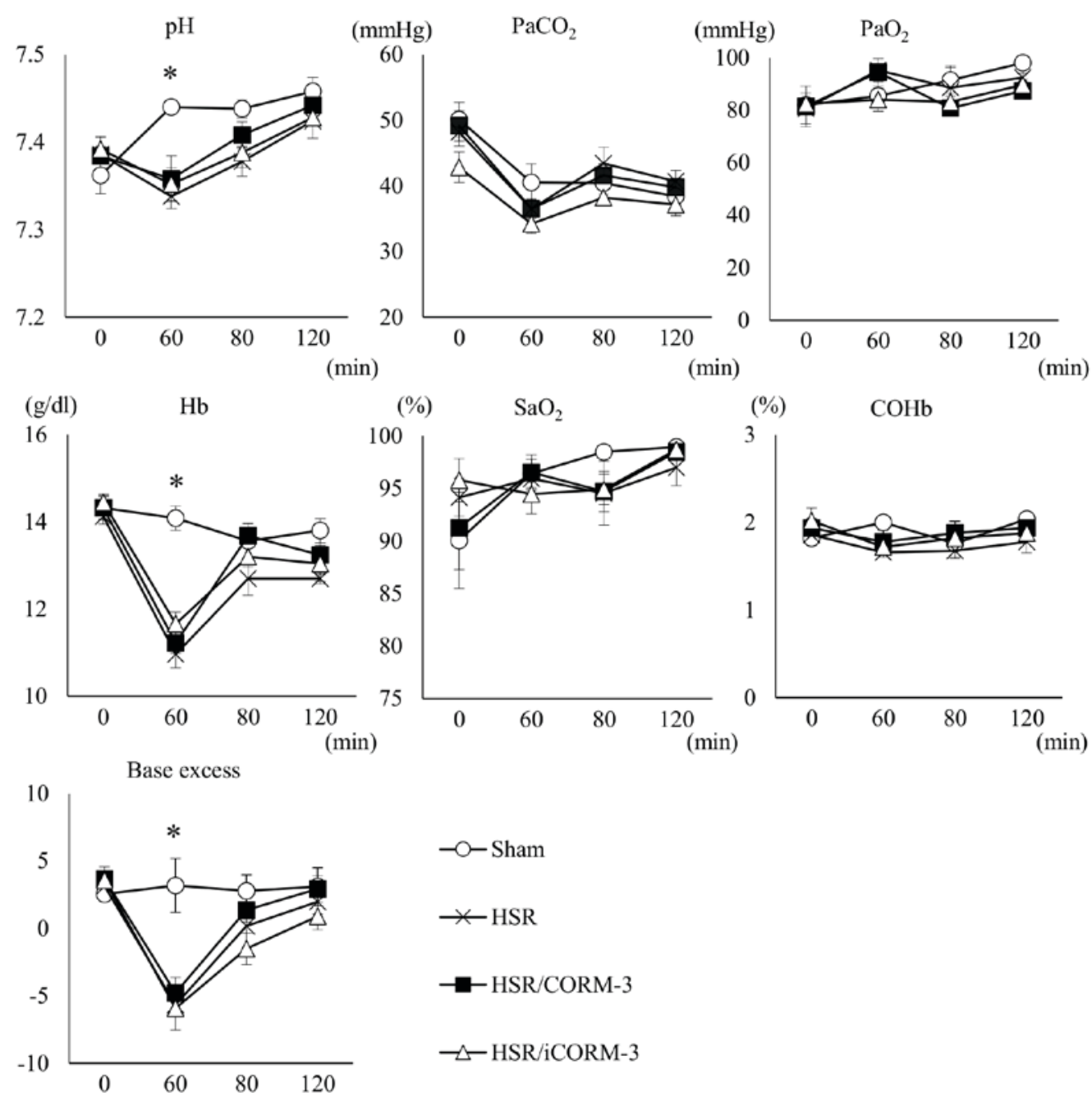

Figure 6. CORM-3 administration following HSR has no influence on blood carboxyhemoglobin levels and preserves oxygenation, as measured by ABG analysis, during HSR. Arterial blood gas (ABG) analysis was performed at the following time points during HSR: 0 min, at the beginning of the hemorrhagic shock; $60 \mathrm{~min}$, at the end of the hemorrhagic shock; $80 \mathrm{~min}$, at $5 \mathrm{~min}$ after the completion of blood reinfusion and the subsequent drug administration; $120 \mathrm{~min}$, at the end of the resuscitation. The horizontal axis in each graph indicates the time after the beginning of the hemorrhagic shock, and the vertical axis shows each parameter of the ABG analysis. Statistical analysis was performed using analysis of variance followed by the Tukey-Kramer multiple comparisons method among groups. Data are presented as the mean $\pm \mathrm{SEM}$ ( $\mathrm{n}=5$ for each group). ${ }^{*} \mathrm{P}<0.05$ (sham vs. HSR, HSR/CORM-3, and HSR $/ \mathrm{iCORM}-3$ at 60 min), ABG, arterial blood gas; $\mathrm{COHb}$, carboxyhemoglobin; CORM-3, carbon monoxide-releasing molecule-3; iCORM-3, inactive carbon monoxide-releasing molecule-3; Hb, hemoglobin; HSR, hemorrhagic shock and resuscitation; $\mathrm{PaCO}_{2}$, partial pressure of carbon dioxide; $\mathrm{PaO}_{2}$, partial pressure of oxygen; $\mathrm{SaO}$, saturation of arterial blood oxygen; SEM, standard error of the mean.

study iCORM-3 that still contained ruthenium as the transition metal but lost the capability to release CO. This drug did not ameliorate HSR-induced lung injury, did not exert any effects on the expression of either pro- or anti-inflammatory cytokines, and did not influence lung vascular permeability and apoptosis parameters. Thus, it is considered that the therapeutic effect of CORM-3 demonstrated in the present study is dependent on the CO released by CORM-3. Second, we did not investigate the effect of CORM-3 in organ tissues other than the lung. Previous studies indicated therapeutic effects of CORM-3 on the liver, the kidneys, and the heart, among others $(26,29,60)$. However, our previous study revealed that the established HSR procedure mainly injured the lung, and only to a lesser extent the liver and the kidneys (34). Thus, the beneficial effects of CORM-3 in the present study are less likely to be based on an indirect modification of the lung by changes in other organs.
Third, we showed that CORM-3 did not have any detrimental effect on the ABG analysis and the vital signs during HSR. However, long-term effects or unwanted effects in other organs including the brain, liver, and kidneys were not investigated. Since CORM-3 is a ruthenium-based complex, a possible accumulation of ruthenium after $\mathrm{CO}$ liberation could result in undesired effects. Further studies are needed to investigate the long-term safety of CORM-3 in various organs.

In conclusion, the HSR procedure caused acute lung injury in a rat model, as demonstrated by the upregulation of proinflammatory mediators, neutrophil migration into the lung, and pulmonary edema, which led to apoptotic cell death. CORM-3 administration immediately after HSR ameliorated the HSR-induced lung injury via suppression of proinflammatory cytokines, induction of IL-10 gene expression, and reduction of apoptotic cell death. In addition, we did not observe 
any detrimental effect on the ABG analysis and vital signs during HSR. These findings suggest that CORM-3 treatment ameliorated HSR-induced lung injury, at least in part, through anti-inflammatory and anti-apoptotic effects. Although further studies are needed to clarify the precise mechanism and pharmacological features of CORM-3, CORM-3 administration may be a promising therapeutic approach for acute lung injury after HSR.

\section{Acknowledgements}

The authors would like to thank Dr Reiko Akagi (Yasuda Women's University, Hiroshima, Japan) for providing cDNAs of TNF and iNOS. The authors would also like to thank Dr Akihiko Taniguchi (Okayama University Graduate School of Medicine, Dentistry, and Pharmaceutical Sciences, Okayama, Japan) for technical support of BAL analysis.

\section{Funding}

This study was supported by the Japan Society for the Promotion of Science (JSPS) Grant-in-Aid for Scientific Research (KAKENHI) Grant no. JP16K10972.

\section{Availability of data and materials}

The dataset used and/or analyzed during the current study are available from the corresponding author on reasonable request.

\section{Authors' contributions}

YK, TT, HS, KI, and HM contributed to the conception and design of the study. YK, RN, EO, and HS performed experiments and collected data. YK, HS, KI, and TT analyzed and interpreted the data. YK drafted and wrote the manuscript. TT and HM revised the manuscript critically for important intellectual content. HM supervised the study and gave final approval of the version to be published. All authors approved the final version of the manuscript.

\section{Ethics approval and consent to participate}

The present study was approved by the Animal Use and Care Committee of the Okayama University Medical School (OKU-2015429) on September 2, 2015. The care and handling of the animals were conducted in accordance with the National Institutes of Health guidelines for Animal Research. The present study also conformed to guidelines for the care and use of laboratory animals that followed the ARRIVE (Animal Research: Reporting of In Vivo Experiments) guidelines and the 2013 AVMA euthanasia guidelines.

\section{Patient consent for publication}

Not applicable.

\section{Competing interests}

The authors declare that they have no competing interests.

\section{References}

1. Dewar D, Moore FA, Moore EE and Balogh Z: Postinjury multiple organ failure. Injury 40: 912-918, 2009.

2. Ciesla DJ, Moore EE, Johnson JL, Cothren CC, Banerjee A, Burch JM and Sauaia A: Decreased progression of postinjury lung dysfunction to the acute respiratory distress syndrome and multiple organ failure. Surgery 140: 640-648, 2006.

3. Ware LB: Pathophysiology of acute lung injury and the acute respiratory distress syndrome. Semin Respir Crit Care Med 27: 337-349, 2006.

4. ARDS Definition Task Force; Ranieri VM, Rubenfeld GD, Thompson BT, Ferguson ND, Caldwell E, Fan E, Camporota L and Slutsky AS: Acute respiratory distress syndrome: The berlin definition. JAMA 307: 2526-2533, 2012.

5. Acute Respiratory Distress Syndrome Network; Brower RG, Matthay MA, Morris A, Schoenfeld D, Thompson BT and Wheeler A: Ventilation with lower tidal volumes as compared with traditional tidal volumes for acute lung injury and the acute respiratory distress syndrome. N Engl J Med 342: 1301-1308, 2000.

6. Tonelli AR, Zein J, Adams J and Ioannidis JP: Effects of interventions on survival in acute respiratory distress syndrome: An umbrella review of 159 published randomized trials and 29 meta-analyses. Intensive Care Med 40: 769-787, 2014.

7. Adhikari N, Burns KE and Meade MO: Pharmacologic therapies for adults with acute lung injury and acute respiratory distress syndrome. Cochrane Database Syst Rev: CD004477, 2004.

8. Peter JV, John P, Graham PL, Moran JL, George IA and Bersten A: Corticosteroids in the prevention and treatment of acute respiratory distress syndrome (ARDS) in adults: Meta-analysis. BMJ 336: 1006-1009, 2008

9. Rose JJ, Wang L, Xu Q, McTiernan CF, Shiva S, Tejero J and Gladwin MT: Carbon monoxide poisoning: Pathogenesis, management, and future directions of therapy. Am J Respir Crit Care Med 195: 596-606, 2017

10. Gullotta F, di Masi A, Coletta M and Ascenzi P: CO metabolism, sensing, and signaling. Biofactors 38: 1-13, 2012

11. Bilban M, Haschemi A, Wegiel B, Chin BY, Wagner O and Otterbein LE: Heme oxygenase and carbon monoxide initiate homeostatic signaling. J Mol Med (Berl) 86: 267-279, 2008.

12. Takahashi T, Shimizu H, Morimatsu H, Maeshima K, Inoue K, Akagi R, Matsumi M, Katayama $\mathrm{H}$ and Morita K: Heme oxygenase-1 is an essential cytoprotective component in oxidative tissue injury induced by hemorrhagic shock. J Clin Biochem Nutr 44: 28-40, 2009.

13. Otterbein LE, Mantell LL and Choi AM: Carbon monoxide provides protection against hyperoxic lung injury. Am J Physiol 276: L688-L694, 1999.

14. Sarady JK, Zuckerbraun BS, Bilban M, Wagner O, Usheva A, Liu F, Ifedigbo E, Zamora R, Choi AM and Otterbein LE: Carbon monoxide protection against endotoxic shock involves reciprocal effects on iNOS in the lung and liver. FASEB J 18: 854-856, 2004.

15. Song R, Kubo M, Morse D, Zhou Z, Zhang X, Dauber JH, Fabisiak J, Alber SM, Watkins SC, Zuckerbraun BS, et al: Carbon monoxide induces cytoprotection in rat orthotopic lung transplantation via anti-inflammatory and anti-apoptotic effects. Am J Pathol 163: 231-242, 2003.

16. Zhang X, Shan P, Otterbein LE, Alam J, Flavell RA, Davis RJ, Choi AM and Lee PJ: Carbon monoxide inhibition of apoptosis during ischemia-reperfusion lung injury is dependent on the p38 mitogen-activated protein kinase pathway and involves caspase 3 . J Biol Chem 278: 1248-1258, 2003.

17. Zuckerbraun BS, Mccloskey CA, Gallo D, Liu F, Ifedigbo E, Otterbein LE and Billiar TR: Carbon monoxide prevents multiple organ injury in a model of hemorrhagic shock and resuscitation. Shock 23: 527-532, 2005.

18. Kawanishi S, Takahashi T, Morimatsu H, Shimizu H, Omori E, Sato K, Matsumi M, Maeda S, Nakao A and Morita K: Inhalation of carbon monoxide following resuscitation ameliorates hemorrhagic shock-induced lung injury. Mol Med Rep 7: 3-10, 2013.

19. Kanagawa F, Takahashi T, Inoue K, Shimizu H, Omori E, Morimatsu H, Maeda S, Katayama H, Nakao A and Morita K: Protective effect of carbon monoxide inhalation on lung injury after hemorrhagic shock/resuscitation in rats. J Trauma 69: 185-194, 2010.

20. Gorman D, Drewry A, Huang YL and Sames C: The clinical toxicology of carbon monoxide. Toxicology 187: 25-38, 2003.

21. Motterlini R, Clark JE, Foresti R, Sarathchandra P, Mann BE and Green CJ: Carbon monoxide-releasing molecules: Characterization of biochemical and vascular activities. Circ Res 90: e17-e24, 2002. 
22. Motterlini R, Mann BE, Johnson TR, Clark JE, Foresti R and Green CJ: Bioactivity and pharmacological actions of carbon monoxide-releasing molecules. Curr Pharm Des 9: 2525-2539, 2003.

23. Motterlini R and Otterbein LE: The therapeutic potential of carbon monoxide. Nat Rev Drug Discov 9: 728-743, 2010.

24. Motterlini R: Carbon monoxide-releasing molecules (CO-RMs) Vasodilatory, anti-ischaemic and anti-inflammatory activities. Biochem Soc Trans 35: 1142-1146, 2007.

25. Foresti R, Bani-Hani MG and Motterlini R: Use of carbon monoxide as a therapeutic agent: Promises and challenges. Intensive Care Med 34: 649-658, 2008.

26. Guo Y, Stein AB, Wu WJ, Tan W, Zhu X, Li QH, Dawn B, Motterlini R and Bolli R: Administration of a CO-releasing molecule at the time of reperfusion reduces infarct size in vivo. Am J Physiol Heart Circ Physiol 286: H1649-H1653, 2004.

27. De Backer O, Elinck E, Blanckaert B, Leybaert L, Motterlini R and Lefebvre RA: Water-soluble CO-releasing molecules reduce the development of postoperative ileus via modulation of MAPK/HO-1 signalling and reduction of oxidative stress. Gut 58 347-356, 2009.

28. Fredenburgh LE, Kraft BD, Hess DR, Harris RS, Wolf MA, Suliman HB, Roggli VL, Davies JD, Winkler T, Stenzler A, et al: Effects of inhaled $\mathrm{CO}$ administration on acute lung injury in baboons with pneumococcal pneumonia. Am J Physiol Lung Cell Mol Physiol 309: L834-L846, 2015.

29. Nassour I, Kautza B, Rubin M, Escobar D, Luciano J, Loughran P, Gomez H, Scott J, Gallo D, Brumfield J, et al: Carbon monoxide protects against hemorrhagic shock and resuscitation-induced microcirculatory injury and tissue injury. Shock 43: 166-171, 2015.

30. Kilkenny C, Browne WJ, Cuthill IC, Emerson M and Altman DG: Improving bioscience research reporting: The ARRIVE guidelines for reporting animal research. Osteoarthritis Cartilage 20: 256-260, 2012

31. American Veterinary Medical Association: AVMA guidelines for the euthanasia of animals: 2013 edition. https://www.avma org/KB/Policies/Documents/euthanasia.pdf. Accessed August 4, 2018.

32. Foresti R, Hammad J, Clark JE, Johnson TR, Mann BE, Friebe A, Green CJ and Motterlini R: Vasoactive properties of CORM-3, a novel water-soluble carbon monoxide-releasing molecule. $\mathrm{Br}$ J Pharmacol 142: 453-460, 2004.

33. Inoue K, Takahashi T, Uehara K, Shimuzu H, Ido K, Morimatsu H, Omori E, Katayama H, Akagi R and Morita K: Protective role of heme oxygenase 1 in the intestinal tissue injury in hemorrhagic shock in rats. Shock 29: 252-261, 2008.

34. Maeshima K, Takahashi T, Uehara K, Shimizu H, Omori E, Yokoyama M, Tani T, Akagi R and Morita K: Prevention of hemorrhagic shock-induced lung injury by heme arginate treatment in rats. Biochem Pharmacol 69: 1667-1680, 2005.

35. Murakami K, McGuire R, Cox RA, Jodoin JM, Bjertnaes LJ, Katahira J, Traber LD, Schmalstieg FC, Hawkins HK, Herndon DN and Traber DL: Heparin nebulization attenuates acute lung injury in sepsis following smoke inhalation in sheep. Shock 18: 236-241, 2002.

36. Zegdi R, Fabre O, Cambillau M, Fornès P, Tazi KA, Shen M, Hervé P, Carpentier A and Fabiani JN: Exhaled nitric oxide and acute lung injury in a rat model of extracorporeal circulation. Shock 20: 569-574, 2003.

37. Jiang H, Meng F, Li W, Tong L, Qiao H and Sun X: Splenectomy ameliorates acute multiple organ damage induced by liver warm ischemia reperfusion in rats. Surgery 141: 32-40, 2007.

38. Stephens KE, Ishizaka A, Larrick JW and Raffin TA: Tumor necrosis factor causes increased pulmonary permeability and edema. Comparison to septic acute lung injury. Am Rev Respir Dis 137: 1364-1370, 1988

39. Wohlauer M, Moore EE, Silliman CC, Fragoso M, Gamboni F, Harr J, Accurso F, Wright F, Haenel J, Fullerton D and Banerjee A: Nebulized hypertonic saline attenuates acute lung injury following trauma and hemorrhagic shock via inhibition of matrix metalloproteinase-13. Crit Care Med 40: 2647-2653, 2012.

40. Kosaka J, Morimatsu H, Takahashi T, Shimizu H, Kawanishi S, Omori E, Endo Y,Tamaki N, Morita M and Morita K: Effects of biliverdin administration on acute lung injury induced by hemorrhagic shock and resuscitation in rats. PLoS One 8: e63606, 2013.

41. Nakahira K, Takahashi T, Shimizu H, Maeshima K, Uehara K, Fujii H, Nakatsuka H, Yokoyama M, Akagi R and Morita K Protective role of heme oxygenase-1 induction in carbon tetrachloride-induced hepatotoxicity. Biochem Pharmacol 66: 1091-1105, 2003.
42. Umeda K, Takahashi T, Inoue K, Shimizu H, Maeda S, Morimatsu H, Omori E, Akagi R, Katayama $\mathrm{H}$ and Morita $\mathrm{K}$ : Prevention of hemorrhagic shock-induced intestinal tissue injury by glutamine via heme oxygenase-1 induction. Shock 31: 40-49, 2009.

43. Kurimoto E, Miyahara N, Kanehiro A, Waseda K, Taniguchi A, Ikeda G, Koga H, Nishimori H, Tanimoto Y, Kataoka M, et al: IL-17A is essential to the development of elastase-induced pulmonary inflammation and emphysema in mice. Respir Res 14: 5, 2013.

44. Yamaoka M, Shimizu H, Takahashi T, Omori E and Morimatsu H Dynamic changes in Bach1 expression in the kidney of rhabdomyolysis-associated acute kidney injury. PLoS One 12: e0180934, 2017.

45. Rushing GD and Britt LD: Reperfusion injury after hemorrhage: A collective review. Ann Surg 247: 929-937, 2008.

46. Alshehri A, Bourguignon MP, Clavreul N, Badier-Commander C, Gosgnach W, Simonet S, Vayssettes-Courchay C, Cordi A, Fabiani JN, Verbeuren TJ and Félétou M: Mechanisms of the vasorelaxing effects of CORM-3, a water-soluble carbon monoxide-releasing molecule: Interactions with eNOS. Naunyn Schmiedebergs Arch Pharmacol 386: 185-196, 2013.

47. Shimzu K, Takahashi T, Iwasaki T, Shimizu H, Inoue K, Morimatsu H, Omori E, Matsumi M, Akagi R and Morita K: Hemin treatment abrogates monocrotaline-induced pulmonary hypertension. Med Chem 4: 572-576, 2008.

48. Tamion F, Richard V, Bonmarchand G, Leroy J, Lebreton J and Thuillez C: Induction of heme-oxygenase-1 prevents the systemic responses to hemorrhagic shock. Am J Respir Crit Care Med 164: 1933-1938, 2001

49. Otterbein LE, Otterbein SL, Ifedigbo E, Liu F, Morse DE, Fearns C, Ulevitch RJ, Knickelbein R, Flavell RA and Choi AM: MKK3 mitogen-activated protein kinase pathway mediates carbon monoxide-induced protection against oxidant-induced lung injury. Am J Pathol 163: 2555-2563, 2003.

50. Zhang X, Shan P, Alam J, Fu X and Lee PJ: Carbon monoxide differentially modulates STAT1 and STAT3 and inhibits apoptosis via a phosphatidylinositol 3-kinase/Akt and p38 kinase-dependent STAT3 pathway during anoxia-reoxygenation injury. J Biol Chem 280: 8714-8721, 2005

51. DolinayT,Szilasi M,Liu Mand Choi AM:Inhaled carbon monoxide confers antiinflammatory effects against ventilator-induced lung injury. Am J Respir Crit Care Med 170: 613-620, 2004.

52. Koulouras VP, Li R, Chen L and Hedenstierna GG: Effects of inhaled carbon monoxide and glucocorticoids in porcine endotoxin sepsis. Int J Clin Exp Med 4: 53-66, 2011.

53. Jung SS, Moon JS, Xu JF, Ifedigbo E, Ryter SW, Choi AM and Nakahira K: Carbon monoxide negatively regulates NLRP3 inflammasome activation in macrophages. Am J Physiol Lung Cell Mol Physiol 308: L1058-L1067, 2015.

54. Kim SK, Joe Y, Chen Y, Ryu J, Lee JH, Cho GJ, Ryter SW and Chung HT: Carbon monoxide decreases interleukin-1 $\beta$ levels in the lung through the induction of pyrin. Cell Mol Immunol 14: 349-359, 2017

55. Queiroga CS, Almeida AS and Vieira HL: Carbon monoxide targeting mitochondria. Biochem Res Int 2012: 749845, 2012.

56. Gomez H, Kautza B, Escobar D, Nassour I, Luciano J, Botero AM, Gordon L, Martinez S, Holder A, Ogundele O, et al: Inhaled carbon monoxide protects against the development of shock and mitochondrial injury following hemorrhage and resuscitation. PLoS One 10: e0135032, 2015.

57. Sun B, Sun H, Liu C, Shen J, Chen Z and Chen X: Role of CO-releasing molecules liberated $\mathrm{CO}$ in attenuating leukocytes sequestration and inflammatory responses in the lung of thermally injured mice. J Surg Res 139: 128-135, 2007.

58. Qin W, Zhang J, Lv W, Wang X and Sun B: Effect of carbon monoxide-releasing molecules II-liberated $\mathrm{CO}$ on suppressing inflammatory response in sepsis by interfering with nuclear factor kappa B activation. PLoS One 8: e75840, 2013.

59. Taylor BS and Geller DA: Molecular regulation of the human inducible nitric oxide synthase (iNOS) gene. Shock 13: 413-424, 2000.

60. Tayem Y, Johnson TR, Mann BE, Green CJ and Motterlini R: Protection against cisplatin-induced nephrotoxicity by a carbon monoxide-releasing molecule. Am J Physiol Renal Physiol 290: F789-F794, 2006.

This work is licensed under a Creative Commons Attribution-NonCommercial-NoDerivatives 4.0 International (CC BY-NC-ND 4.0) License. 